Monatsschr Kinderheilkd

https://doi.org/10.1007/s00112-021-01409-0

Eingegangen: 3. März 2021

Angenommen: 14. Juni 2021

๑) Der/die Autor(en) 2022

\section{Redaktion}

Berthold Koletzko, München

Thomas Lücke, Bochum

Ertan Mayatepek, Düsseldorf

Norbert Wagner, Aachen

Stefan Wirth, Wuppertal

Fred Zepp, Mainz

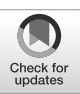

\title{
Charakteristika und klinische Manifestation katheterassoziierter Thrombosen bei Frühgeborenen
}

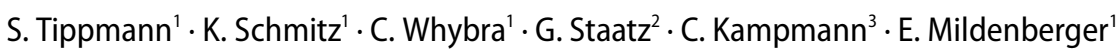 \\ ${ }^{\prime}$ Neonatologie, Zentrum für Kinder - und Jugendmedizin der Unimedizin Mainz, Mainz, Deutschland \\ ${ }^{2}$ Kinderradiologie, Klinik und Poliklinik für Diagnostische und Interventionelle Radiologie, \\ Universitätsmedizin Mainz der Johannes Gutenberg-Universität Mainz, Mainz, Deutschland \\ ${ }^{3}$ Kinderkardiologie, Zentrum für Kinder- und Jugendmedizin, Universitätsmedizin der Johannes \\ Gutenberg-Universität Mainz, Mainz, Deutschland
}

Zusammenfassung

Hintergrund: In der Neonatologie sind peripher eingeführte zentrale Katheter ein häufiger Zugangsweg zur parenteralen Ernährung und Verabreichung von Medikamenten und Flüssigkeit. Die Vorteile stehen den Risiken wie Infektion, Thrombose und Fehllage gegenüber.

Fragestellung: Welche Charakteristika und klinischen Zeichen weisen auf das Vorliegen einer katheterassoziierten Thrombose hin?

Material und Methoden: In einer retrospektiven Betrachung aus dem Zeitraum 2010-2016 wurden alle Fälle von katheterassoziierten Thrombosen untersucht. Identifiziert wurden 10 Frühgeborene, deren Indikation zur Katheteranlage, Zugangsweg, Lage der Katheterspitze, Liegedauer, klinische Symptome und Krankheitsverlauf analysiert wurden.

Ergebnisse: Bei 10 Frühgeborenen mit einem Gestationsalter von $23+4$ bis $34+5$ SSW wurden 11 Thromboseereignisse beobachtet. Zehn der 11 Thromboseereignisse betrafen die V. cava inferior nach Katheteranlage an der unteren Extremität. Die Indikation zur Katheteranlage waren parenterale Ernährung und chirurgische Eingriffe. Bei 9 Patienten traten perinatale Komplikationen auf. Bei allen Patienten bestand während der Liegedauer der Katheter der Verdacht auf eine Infektion. Das Auftreten der Thrombose wurde nach 3 bis 27 Tagen sonographisch dokumentiert. In 8 Thromboseereignissen war eine Thrombozytopenie, in 4 Fällen eine Beinschwellung auffällig.

Diskussion: Das Patientenkollektiv zeigt Gemeinsamkeiten im klinischen Verlauf und bei den Symptomen, welche auf das Vorliegen einer Thrombose deuten. Auffällig ist außerdem, dass die meisten Thromboseereignisse das Stromgebiet der V. cava inferior betrafen.

\section{Schlüsselwörter}

Peripher eingeführte Katheter · Komplikationen · Thrombose · Thrombozytopenie · Vena cava inferior

\section{Hintergrund}

In der Neonatologie sind peripher eingeführte zentrale Katheter ein häufig genutzter Zugangsweg zur parenteralen Ernährung und zur Verabreichung von Medikamenten und Flüssigkeit. Die Anlage der Katheter erfolgt über periphere Venen, wie die V. (Vena) mediana cubiti an der oberen Extremität und die V. saphena magna an der unteren Extremität. Dabei wird der Katheter über einen peripheren i.v.Zugang durch manuellen Vorschub nach zentralvenös eingebracht. Ziel ist es, die Katheterspitze in der V. cava superior bei Anlage an der oberen Extremität bzw. am 
Übergang zwischen der V. cava inferior und dem rechten Vorhof bei Anlage an der unteren Extremität zu positionieren.

Diese minimal-invasive Möglichkeit ist v.a. bei extrem unreifen Frühgeborenen etabliert [27]. Die Vorteile gegenüber peripheren Venenkathetern sind die reduzierte Anzahl venöser Punktionen („minimal handling "), längere Liegedauer, Möglichkeit der Verabreichung hochkonzentrierter Lösungen und die sichere Verfügbarkeit eines venösen Zugangswegs. Demgegenüber stehen Risiken wie Katheterinfektion, Thrombose, Extravasat, Fehllage und Katheterbrüche $[22,27]$. Die Inzidenz von katheterassoziierten Thrombosen bei Frühgeborenen ist bisher nicht bekannt. Bei reifen Neugeborenen und älteren Kindern werden Thrombosen bei zentralen Kathetern (peripher eingeführte und zentral gelegte Katheter) mit 0,5-1\% der Komplikationen beschrieben $[15,23]$.

\section{Die Entstehung der Thrombose ist noch nicht geklärt}

Der genaue Mechanismus der Thromboseentstehung bei liegendem Katheter ist bisher nicht vollständig geklärt. Zur Beschreibung der Pathogenese wird die Virchow-Trias, bestehend aus Gefäßwandläsion (z. B. Verletzungen der Gefäßintima durch den Katheter selbst), reduzierter Strömungsgeschwindigkeit (z.B. Verlangsamung des Blutflusses bei hohem intraabdominellen Druck) und Hyperkoagulabilität (z.B. durch die infundierten Lösungen und Infektionen), auch heute noch herangezogen $[6,8]$.

Eine nichtzentrale Lage des Katheters, Infektionen und eine lange Liegedauer sind mit der Entstehung von Thrombosen assoziiert [23]. Allein schon das Vorhandensein eines venösen Katheters begünstigt die Entstehung einer Thrombose [13]. Weiterhin wird vermutet, dass der venöse Rückstrom durch eine Verletzung von Venenklappen ungünstig beeinflusst wird [4]. Auch individuelle Faktoren, wie der klinische Zustand des Patienten und eine genetische Prädisposition bezüglich einer Thromboseneigung, erscheinen wichtig [9]. Es ist bisher nicht untersucht, ob das Entstehen einer katheterassoziierten Thrombose von dem Anlageort des Kathe- ters (obere vs. untere Extremität) abhängig ist.

\section{Fragestellungen}

Welche Charakteristika und klinischen Zeichen weisen auf das Vorliegen einer katheterassoziierten Thrombose hin? Finden sich Gemeinsamkeiten im klinischen Verlauf der betroffenen Frühgeborenen in unserem Patientenkollektiv?

\section{Material und Methoden}

In einer retrospektiven Betrachtung am Zentrum für Kinder- und Jugendmedizin der Universitätsmedizin der Johannes Gutenberg-Universität Mainz wurden für die Jahre 2010-2016 alle Fälle von katheterassoziierten Thrombosen analysiert. Das klinikinterne Patientenerfassungssystem wurde nach folgenden Diagnosen durchsucht: Thrombose, Embolie, Phlebitis, Thrombophlebitis, intrakardiale Thrombose. Die Suche wurde dann auf Früh- und Neugeborene eingegrenzt, und ausschließlich die Thrombosen, welche während der Liegezeit eines peripher eingeführten zentralen Katheters aufgetreten waren, wurden in die Betrachtung einbezogen.

Bei den Früh- und Neugeborenen mit katheterassoziierten Thrombosen wurden folgende Parameter untersucht: Indikation zur Anlage eines peripher eingeführten zentralen Katheters, Zugangsweg (obere oder untere Extremität), Anzahl der Korrekturen, die im Rahmen der Katheteranlage notwendig waren, Lage der Katheterspitze nach endgültiger Fixierung, Liegedauer des Katheters sowie die Osmolarität der infundierten Lösungen. In die Analyse floss mit ein, durch welche Symptomatik die Thrombosen entdeckt wurden, und ob es während der Liegezeit des Katheters Komplikationen, wie z.B. chirurgische Interventionen oder Infektionen, gab, welche Therapie initiiert wurde und ob Residualbefunde verblieben.

\section{Ergebnisse}

Im Studienzeitraum erfüllten 188 Fälle die Suchkriterien. Bei 15 Patienten handelte es sich um Früh- und Neugeborene, davon wurden 10 Patienten mit einer kathe- terassoziierten Thrombose bei liegendem Einschwemmkatheter identifiziert. Im Studienzeitraum wurden in unserer neonatologischen Intensivstation insgesamt 386 Frühgeborene mit einem Geburtsgewicht unter $1500 \mathrm{~g}$ betreut.

Analysiert wurden 11 Thromboseereignisse bei 7 männlichen und 3 weiblichen Patienten. Ein Patient hatte sowohl eine Thrombose der V. cava inferior als auch, bei erneuter Katheteranlage vom linken Arm aus, eine Thrombose der V. subclavia links erlitten.

Die Indikation für den peripher eingeführten zentralen Katheter war bei 8 der 10 Patienten die Verabreichung einer parenteralen Ernährung. Bei 2 von 10 Patienten wurde der Katheter im Rahmen einer Duktusligatur etabliert (• Tab. 1).

Von den 11 beobachteten Thromboseereignissen bei 10 Patienten waren 10 Thrombosen im Bereich der V. cava inferior aufgetreten. 9 von 10 Patienten waren extrem unreife Frühgeborene mit einem Gestationsalter zwischen $23+4$ SSW und $27+0$ SSW sowie einem Geburtsgewicht zwischen $330 \mathrm{~g}$ und $1040 \mathrm{~g}$. Ein Patient war ein Frühgeborenes von $34+5$ SSW mit Geburtsgewicht von $2180 \mathrm{~g}$, mit der Diagnose einer Trisomie 21 und perinataler Asphyxie und kompliziertem postnatalen Verlauf.

Bei einem Patienten war eine mehrfache Lagekorrektur des Katheters notwendig. Bei mindestens 4 Patienten wurde der Katheter im Rahmen der Anlage einmal korrigiert.

Bei allen Patienten lag die Katheterspitze nach der Korrektur initial zentral. In einem Fall wurde im Verlauf die Katheterspitze in der Lebervene dokumentiert. Die Osmolarität der infundierten Lösung (zumeist parenterale Ernährung) betrug immer unter $1800 \mathrm{mosmol} / \mathrm{l}$. Die infundierten Lösungen enthielten keinen Heparinzusatz. Das Erkennen der Thrombose lag in unserem Patientenkollektiv 3 bis 27 Tage nach Einbringen des Katheters. Die erste Symptomatik, welche (retrospektiv) auf das Vorliegen einer Thrombose hindeutete, wurde einen bis 15 Tage vor der Diagnosestellung dokumentiert. Sofern es der Zustand der Patienten zuließ, wurde der mit der Thrombose assoziierte Katheter umgehend entfernt. 


\begin{tabular}{|c|c|c|c|c|c|c|c|c|c|c|}
\hline 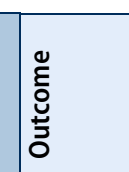 & 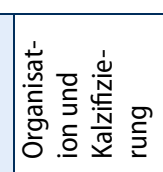 & & 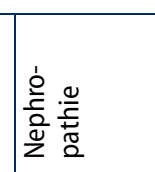 & 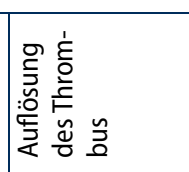 & 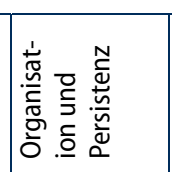 & 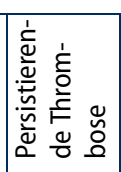 & 竞总 & 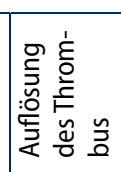 & 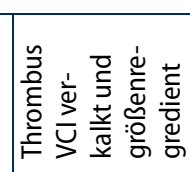 & 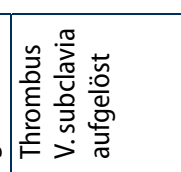 \\
\hline 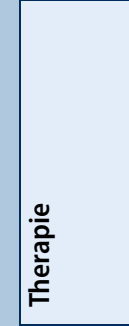 & 崖 & 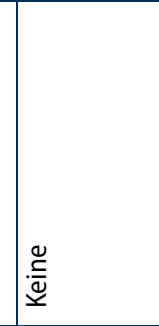 & 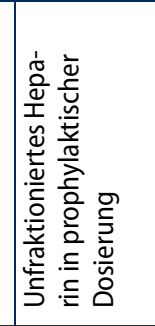 & 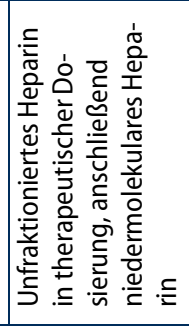 & 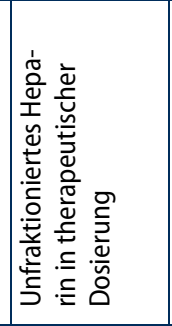 & 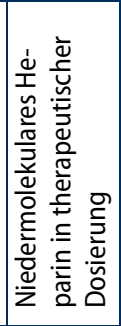 & : & 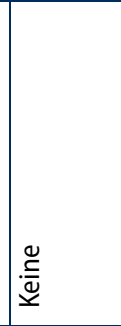 & 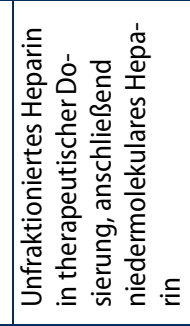 & 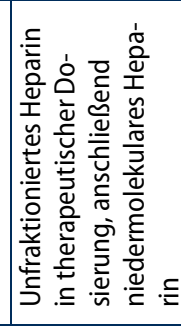 \\
\hline 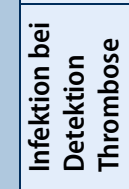 & 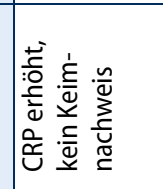 & 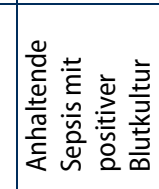 & 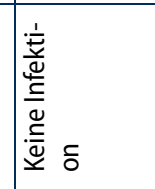 & 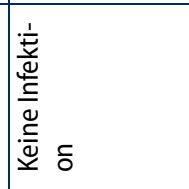 & 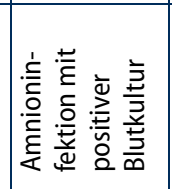 & 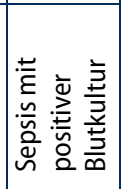 & 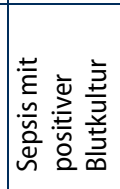 & 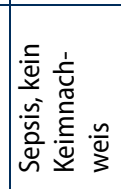 & 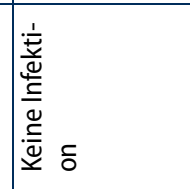 & 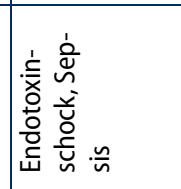 \\
\hline 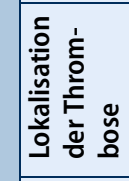 & $\bar{z}$ & $\bar{y}$ & 旁 & 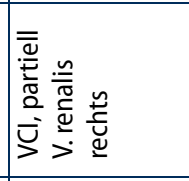 & $\bar{Y}$ & $\bar{Y}$ & $\bar{Y}$ & $\bar{y}$ & $\bar{y}$ & 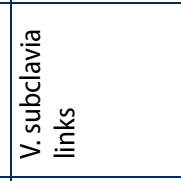 \\
\hline 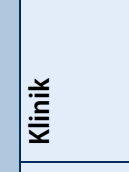 & 竧 & 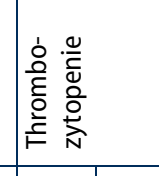 & 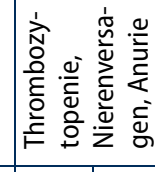 & 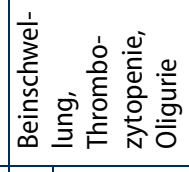 & 总 & 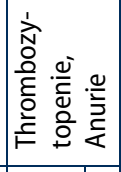 & 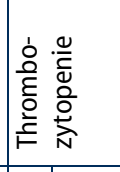 & 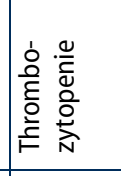 & 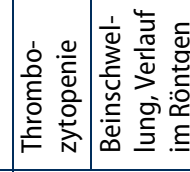 & 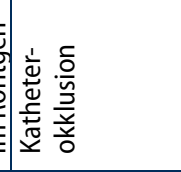 \\
\hline 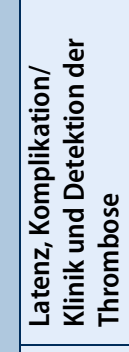 & 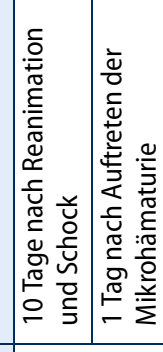 & 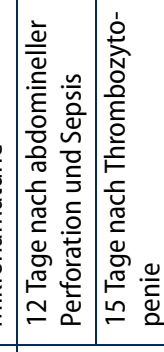 & 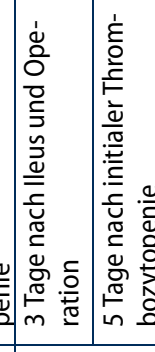 & 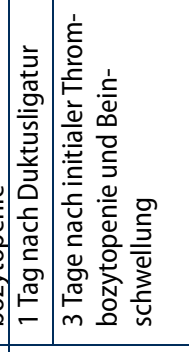 & 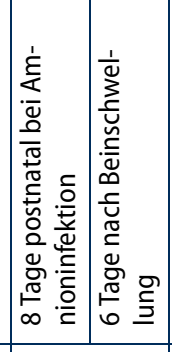 & 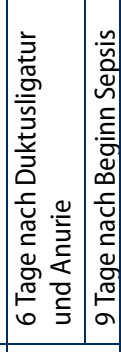 & 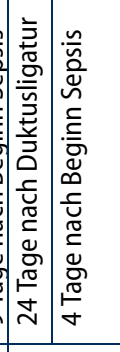 & 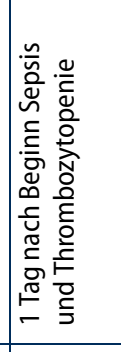 & 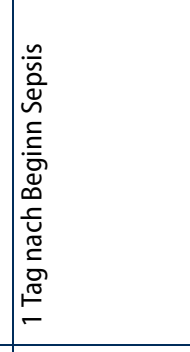 & 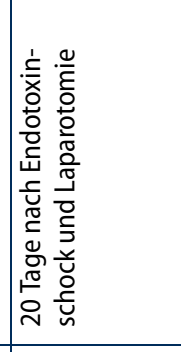 \\
\hline 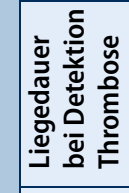 & 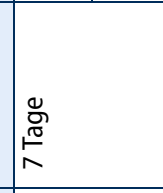 & $\begin{array}{l}\stackrel{\Xi}{\Xi} \\
\stackrel{\Xi}{\simeq} \\
\simeq\end{array}$ & 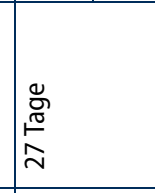 & 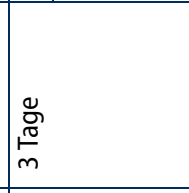 & 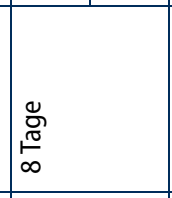 & $\begin{array}{l}\text { 总 } \\
\text { 兽 }\end{array}$ & $\begin{array}{l}\stackrel{a}{\Xi} \\
\stackrel{\Xi}{\simeq} \\
\simeq\end{array}$ & 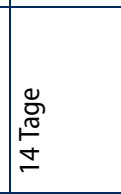 & 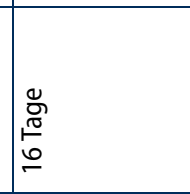 & 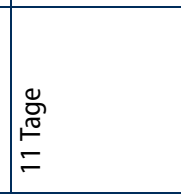 \\
\hline 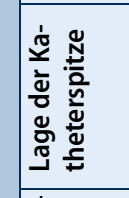 & 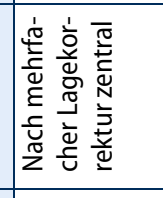 & $\begin{array}{l}\text { T⿱乛ु } \\
\text { 离 } \\
\text { N }\end{array}$ & 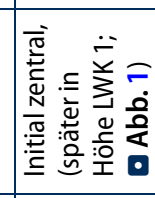 & & 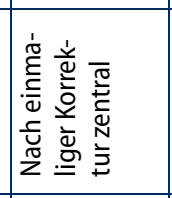 & 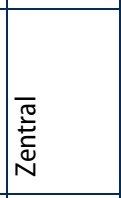 & 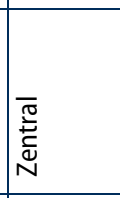 & 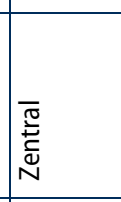 & 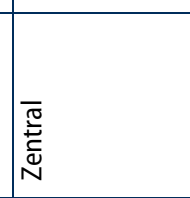 & 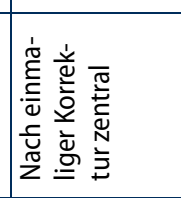 \\
\hline 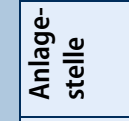 & 离 & 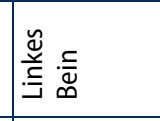 & 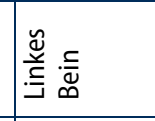 & 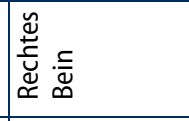 & 离 & 离 & 离 & 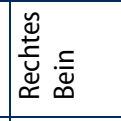 & 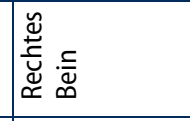 & 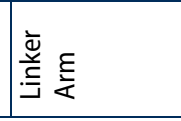 \\
\hline 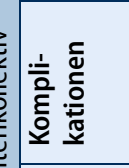 & 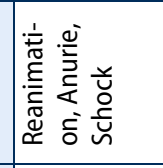 & 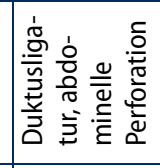 & 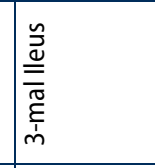 & 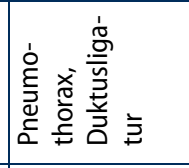 & 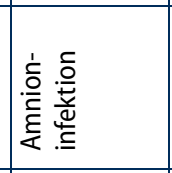 & 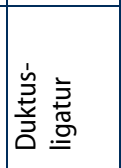 & 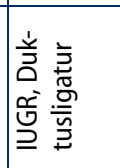 & 产 & \multicolumn{2}{|c|}{ 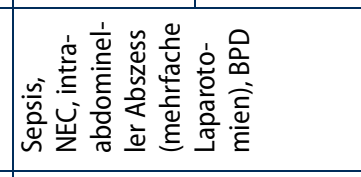 } \\
\hline 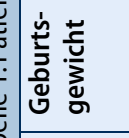 & $\begin{array}{l}0 \\
0 \\
0 \\
0\end{array}$ & 总 & Oे & : & $\stackrel{0}{\stackrel{0}{1}}$ & 票 & 品 & 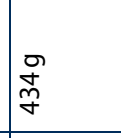 & \multicolumn{2}{|l|}{ Oे } \\
\hline 芯 & $\begin{array}{l}+ \\
+ \\
\stackrel{+}{+}\end{array}$ & 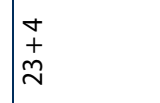 & \begin{tabular}{l}
$\mp$ \\
\multirow{N}{*}{}
\end{tabular} & $\begin{array}{l}\text { 年 } \\
\text { a }\end{array}$ & 足 & $\begin{array}{l}m \\
+ \\
\vdots \\
w\end{array}$ & 齐 & \begin{tabular}{l}
+ \\
\multirow{J}{*}{} \\
\end{tabular} & \multicolumn{2}{|l|}{$\begin{array}{l}\stackrel{0}{+} \\
\stackrel{+}{N}\end{array}$} \\
\hline$=$ & - & N & m & + & in & 0 & $\Lambda$ & $\infty$ & \multicolumn{2}{|l|}{ a } \\
\hline
\end{tabular}




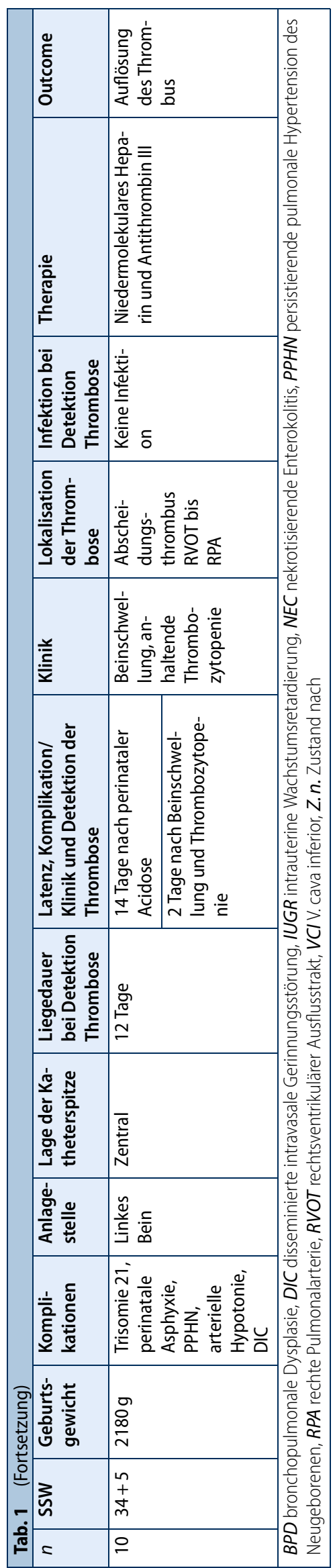

In allen Fällen erfolgte die Diagnosestellung durch Sonographie. Bei 8 Thromboseereignissen war eine persistierende Thrombozytopenie Anlass für die Ultraschalluntersuchung. Bei 4 Patienten trat eine Beinschwellung auf. In einem Fall war eine Mikrohämaturie bei gleichzeitig vorliegender Nierenvenenthrombose auffällig und hatte zur sonographischen Suche nach einer Thrombose geführt. Der Thrombus in der V. subclavia fiel durch eine Katheterokklusion auf. In einem Fall war in einem Röntgenbild ein auffälliger Katheterverlauf hinweisend (• Abb. 1).

Bei 9/10 Patienten waren perinatale Komplikationen aufgetreten. Ein Patient wurde innerhalb der ersten Lebenstage kardiopulmonal reanimiert; ein Patient entwickelte im Rahmen einer perinatalen Asphyxie eine persistierende pulmonale Hypertension. Während der Liegezeit der Katheter erhielten 4/10 Patienten eine Duktusligatur; bei $3 / 10$ Patienten war der Verlauf durch abdominelle Operationen geprägt.

Bei allen 10 Patienten bestand während des Zeitraums des einliegenden Katheters der Verdacht auf eine Infektion bzw. wurde bei Verschlechterung des Allgemeinzustands eine antibakterielle Therapie begonnen. Es zeigte sich allerdings bei keinem mit der Thrombose assoziierten Katheter ein Keimnachweis an der Katheterspitze, auch wenn die Blutkultur in 4 von 10 Fällen während der Katheterliegezeit positiv war.

6 Patienten erhielten aufgrund der Thrombose eine medikamentöse Therapie. 5 Patienten erhielten eine Therapie mit unfraktioniertem Heparin in therapeutischer Dosis. Einem Patienten wurde bei frischer intraventrikulärer Hämorrhagie unfraktioniertes Heparin in prophylaktischer Dosierung verabreicht. Bei 5 dieser Patienten wurde eine Therapie mit niedermolekularem Heparin für 6 Wochen angeschlossen. Ein Patient erhielt zusätzlich repetitiv Antithrombin III. 4 Patienten wurden aufgrund von Kontraindikationen bei schlechtem Allgemeinzustand und anhaltender Thrombozytopenie nicht therapiert.

Konsekutiv war bei 4 Patienten kein Residuum der Thrombose mehr nachweisbar. Bei einem Patienten kam es zu einem vollständigen thrombotischen Verschluss der
V. cava inferior, bei 6 Patienten kam es zur Organisation der Thrombose im Verlauf. Die Thrombose in der V. subclavia war unter Therapie (Fortführung der Therapie mit niedermolekularem Heparin) komplett regredient.

\section{Diskussion und Schlussfolgerung}

In unserem Patientenkollektiv von 10 Frühgeborenen, welche katheterassoziierte Thrombosen entwickelten, zeigen sich Gemeinsamkeiten im klinischen Verlauf. Alle 10 Patienten hatten einen peripher eingeführten zentralen Katheter im Bereich der unteren Extremität und entwickelten eine Thrombose im Stromgebiet der V. cava inferior. Nur ein Patient hatte zudem eine Thrombose in der V. subclavia (nach einliegendem Katheter von der oberen Extremität). Bei allen Patienten hatten klinische Symptome wie Beinschwellung und anhaltende Thrombozytopenie zur Suche nach einer Thrombose geführt. Entdeckt wurde die Thrombose bei allen Frühgeborenen mittels Sonographie.

\section{Risikofaktoren für eine Thromboseentstehung}

Insgesamt scheinen in unserer Betrachtung der Patienten mehrere Faktoren einen Einfluss bei der Thromboseentstehung zu haben.

Unreife. Ein Auftreten von katheterassoziierten Thrombosen bei Frühgeborenen mit kompliziertem postnatalem Verlauf und operativen Eingriffen, vergleichbar mit unserem Patientenkollektiv (•Tab. 1), findet sich auch in der Literatur (- Tab. 2; [16]). Während die Meinung gilt, dass katheterassoziierte Komplikationen im Zusammenhang mit Unreife und Allgemeinzustand des Kindes stehen, gibt es ebenfalls Auswertungen, die keinen Zusammenhang zwischen katheterassoziierten Komplikationen und Unreife dokumentieren $[10,19]$.

Perinatale Komplikationen. Nach stattgehabter Reanimation oder Hypoxie wird in manchen Fällen eine Störung des Gleichgewichts zwischen Blutungsneigung mit disseminierter intravasaler Gerinnung und Thrombozytopenie sowie übermäßiger 


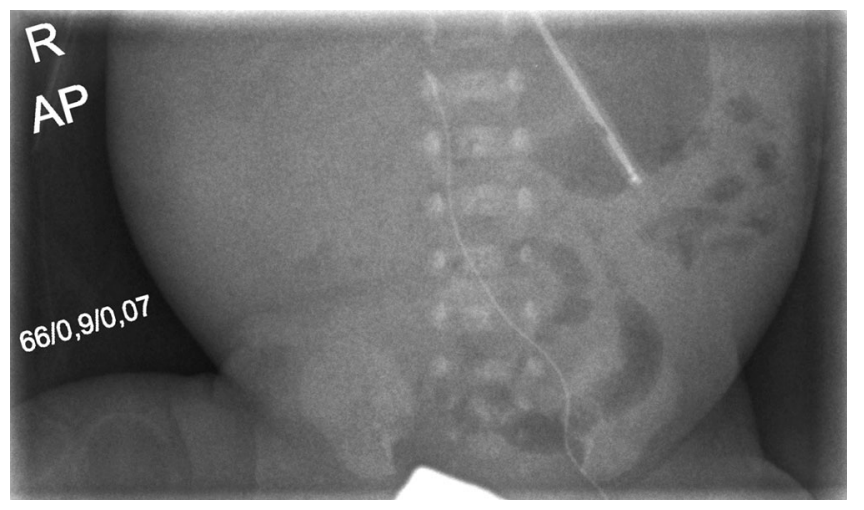

Abb. 1 Fehllage und atypischer, gestauchter Verlauf eines von der unteren Extremität eingebrachten PICC (Patient 3). Der auffällige Katheterverlauf führte zu der sonographischen Untersuchung, in welcher eine Thrombose der V. cava inferior dargestellt wurde
Blutgerinnung mit möglicher Thromboseentstehung beschrieben [2]. Daher ist auch bei unseren Patienten anzunehmen, dass durch stattgehabte Reanimation, Operation oder Schock die Entstehung einer Thrombose begünstigt wurde.

Anlagestelle. In einigen Studien wird ein erhöhtes Thromboserisiko bei Katheteranlage an der unteren Extremität beschrieben - insbesondere, wenn zeitgleich eine abdominale Pathologie vorliegt [16]. Andere Autoren haben keinen Unterschied in der Komplikationsrate nach Katheteranlage von der oberen vs. der unteren Extremität gefunden [1, 6, 20]. Von einer Anlage an der unteren Extremität wird aufgrund gehäuft vorkommender Katheterinfektionen abgeraten [26]. Aufgrund verschiedener anderer Aspekte wird im Gegensatz dazu in einer anderen Publikation wiederum die Anlage eines Katheters von der unteren Extremität aus empfohlen. So seien diese Katheter länger funktionstüchtig und weniger häufig mit Kathetersepsitiden oder Cholestase assoziiert [13]. Von der oberen Extremität aus wird jedoch häufiger über lokale Komplikationen wie Phlebitis und Inflammation und dadurch verkürzte Liegedauer berichtet [26]. In unserer Analyse zeigte sich, dass 10 von 11 Thrombosen bei an der unteren Extremität vorhandenem Katheter aufgefallen sind. Anhand unserer Fallbeispiele lassen sich jedoch keine Rückschlüsse auf den komplikationsloseren Zugangsweg (obere vs. untere Extremität) eines Katheters ziehen.

Anlagekomplikationen. Mehrfachen Anlageversuchen oder mechanischen Veränderungen des Katheters wird ein erhöhtes Thromboserisiko zugeschrieben, da Mani- pulationen mit dem Katheter zu Endothelschäden führen können [24]. Der Endothelschaden kann sekundär die Entstehung einer Thrombose begünstigen. Es wird empfohlen, auch während der Liegedauer mechanische Manipulationen zu vermeiden und den Katheter gut zu fixieren [23]. Insgesamt ist die Dokumentation über Komplikationen während der Katheteranlage sowie während der Liegedauer (beispielsweise Dislozieren während eines Verbandwechsels, erschwerte Anlagebedingungen) unbefriedigend. Hier könnte neben einer Leitlinie zur Katheteranlage auch eine standardisierte Verlaufsdokumentation (Anzahl der Venenpunktion, Lagekorrektur, klinische Auffälligkeiten) hilfreich sein [12]. Eine solche Leitlinie und Dokumentation lagen im Analysezeitraum für unsere Abteilung nicht vor. Grundlage könnten die Empfehlungen der Kommission für Krankenhaushygiene und Infektionsprävention (KRINKO) des Robert KochInstituts sein, wenngleich sich auch hieraus keine eindeutige Richtlinie zur Thromboseprävention bei Früh- und Neugeborenen ableiten lässt $[7,11]$.

Katheterlage. Die zentrale Katheterlage scheint ein wichtiger Punkt bei der Vermeidung von Komplikationen zu sein. Nichtzentrale Katheterlagen führen anscheinend häufig zur frühzeitigen bzw. nichtelektiven Entfernung des Katheters und sind häufiger mit Thrombosen und Sepsitiden assoziiert [8, 14, 27]. Es wird beschrieben, dass die Katheter von der unteren Extremität aus häufiger zentral liegen [3]. Dies gelang in unserer Beobachtungskohorte fast durchgehend. In einem unserer Fälle (Patient 3) war der Katheter im Verlauf nicht mehr zentral positioniert, und der Katheterverlauf radiologisch auffällig. Sonographisch wurde dann die Thrombose nachgewiesen (• Abb. 1).

Liegedauer. Eine lange Liegedauer des Katheters wird mit Komplikationen wie Thrombosen assoziiert $[18,20]$.

Infektionen. Ein eindeutiger Zusammenhang zwischen einer katheterassoziierten Thrombose aufgrund einer Sepsis besteht nicht [25]. Es gibt jedoch Hinweise, dass katheterassoziierte Thrombosen mit einem erhöhten Risiko für Infektionen assoziiert werden können [23]. In unserer Recherche zeigte sich bei keinem mit der Thrombose assoziierten Katheter ein Keimnachweis an der Katheterspitze, auch wenn die Blutkultur in 4 von 10 Fällen während der Katheterliegezeit positiv war.

Therapie. Derzeit gibt es keine Empfehlung bezüglich einer prophylaktischen Heparinisierung zur Vermeidung von katheterassoziierten Thrombosen. Es konnte zwar gezeigt werden, dass es zu einer signifikant längeren Funktionsfähigkeit des Katheters kommt, Thrombosen aber nicht vermieden werden können. Aus infektionspräventiver Sicht wird dies aktuell ebenfalls nicht empfohlen [5, 7, 21]. Bei unserem Kollektiv enthielten die Infusionslösungen keinen Heparinzusatz.

Die Therapieoptionen von Thrombosen bei Früh- und Neugeborenen werden kontrovers diskutiert. In einigen Fällen kann auf eine Therapie verzichtet werden und der spontane Verlauf der Thrombose beobachtet werden. Die Lokalisation, das Ausmaß und die Relevanz der Thrombose sollten ebenso in die Entscheidung miteinbezogen werden, wie weitere Komplikationen und der Allgemeinzustand des Patienten [17]. Therapiekonzepte schließen die Fibrinolyse zur Auflösung des Thrombus, sowie die Antikoagulation zur Vermeidung der weiteren Thrombozytenaggregation ein. In seltenen Fällen kann eine chirurgische Intervention notwendig sein [24]. Unsere Recherche zeigte ein sehr unterschiedliches Outcome mit Auflösung der Thrombose, Organisation oder Persistenz, das unabhängig von der durchgeführten Therapie zu sein schien. Eine Therapieempfehlung lässt sich daher auch unseren Daten nicht herleiten. 
Tab. 2 Literaturübersicht

\begin{tabular}{|c|c|c|c|c|c|c|c|}
\hline Autoren & Titel & Jahr & $\begin{array}{l}\text { Studien- } \\
\text { design }\end{array}$ & $\begin{array}{l}\text { Fallzahl } \\
\text { (PICC) }\end{array}$ & Ergebnis & Schlussfolgerung & Thromboseassoziation \\
\hline $\begin{array}{l}\text { Bashir } \\
\text { et al. [1] }\end{array}$ & $\begin{array}{l}\text { Association between } \\
\text { peripherally inserted } \\
\text { central venous cathe- } \\
\text { ter insertion site and } \\
\text { complication rates in } \\
\text { preterm infants }\end{array}$ & 2016 & $\begin{array}{l}\text { Retro- } \\
\text { spektiv }\end{array}$ & 827 & $\begin{array}{l}\text { Patienten mit Katheter der } \\
\text { unteren Extremität waren } \\
\text { kränker }\end{array}$ & $\begin{array}{l}\text { Kein signifikanter } \\
\text { Unterschied bzgl. } \\
\text { PICC-assoziierter } \\
\text { Komplikationen } \\
\text { zwischen oberer und } \\
\text { unterer Extremität }\end{array}$ & $\begin{array}{l}\text { Keine explizierte Aus- } \\
\text { wertung bezüglich } \\
\text { katheterassoziierter } \\
\text { Thrombosen }\end{array}$ \\
\hline $\begin{array}{l}\text { Van den } \\
\text { Berg et al. } \\
{[3]}\end{array}$ & $\begin{array}{l}\text { Peripherally inserted } \\
\text { central catheter in ex- } \\
\text { tremely preterm infants: } \\
\text { Characteristics and in- } \\
\text { fluencing factors }\end{array}$ & 2017 & $\begin{array}{l}\text { Retro- } \\
\text { spektiv }\end{array}$ & 379 & $\begin{array}{l}\text { Katheter der unteren Extre- } \\
\text { mität hatten häufiger eine } \\
\text { zentrale Lage der Katheter- } \\
\text { spitze und wurden elektiv } \\
\text { entfernt }\end{array}$ & $\begin{array}{l}\text { Auf zentrale Lage der } \\
\text { Katheterspitze ach- } \\
\text { ten, um Komplikati- } \\
\text { onen zu vermeiden }\end{array}$ & $\begin{array}{l}\text { Keine explizierte Aus- } \\
\text { wertung bezüglich } \\
\text { katheterassoziierter } \\
\text { Thrombosen }\end{array}$ \\
\hline $\begin{array}{l}\text { Hoang } \\
\text { et al. [11] }\end{array}$ & $\begin{array}{l}\text { Percutaneously inserted } \\
\text { central catheter for total } \\
\text { parenteral nutrition in } \\
\text { neonates: Complication } \\
\text { rates related to upper } \\
\text { versus lower extremity } \\
\text { insertion }\end{array}$ & 2008 & $\begin{array}{l}\text { Retro- } \\
\text { spektiv }\end{array}$ & 477 & $\begin{array}{l}\text { Weniger Infektionen bei } \\
\text { PICC von unterer Extremi- } \\
\text { tät, weniger häufig Cho- } \\
\text { lestase durch Septikämie }\end{array}$ & $\begin{array}{l}\text { PICC von unterer } \\
\text { Extremität für teilpa- } \\
\text { renterale Ernährung } \\
\text { empfohlen }\end{array}$ & $\begin{array}{l}\text { Keine explizierte Aus- } \\
\text { wertung bezüglich } \\
\text { katheterassoziierter } \\
\text { Thrombosen }\end{array}$ \\
\hline $\begin{array}{l}\text { Kisa et al. } \\
\text { [13] }\end{array}$ & $\begin{array}{l}\text { Major thrombotic com- } \\
\text { plications with lower } \\
\text { limb PICC in surgical } \\
\text { neonates }\end{array}$ & 2015 & $\begin{array}{l}\text { Retro- } \\
\text { spektiv }\end{array}$ & 692 & $\begin{array}{l}\text { Alle Patienten mit Throm- } \\
\text { bose hatten einen Katheter } \\
\text { von der unteren Extremität } \\
\text { mit dezentraler Lage der } \\
\text { Spitze }\end{array}$ & $\begin{array}{l}\text { Auf regelrechte Ka- } \\
\text { theterlage achten } \\
\text { und ggf. Neuanlage } \\
\text { erwägen }\end{array}$ & $\begin{array}{l}\text { Assoziation von } \\
\text { Thrombose und in- } \\
\text { korrekter Katheterlage }\end{array}$ \\
\hline $\begin{array}{l}\text { Ozkiraz } \\
\text { et al. [17] }\end{array}$ & $\begin{array}{l}\text { Peripherally inserted } \\
\text { central venous catheters } \\
\text { in critically ill premature } \\
\text { neonates }\end{array}$ & 2013 & $\begin{array}{l}\text { Retro- } \\
\text { spektiv }\end{array}$ & 68 & $\begin{array}{l}\text { Ernsthafte Komplikationen } \\
\text { bei Frühgeborenen mit kri- } \\
\text { tischem Zustand möglich }\end{array}$ & $\begin{array}{l}\text { Kein Unterschied in } \\
\text { Komplikationsraten } \\
\text { zwischen oberer und } \\
\text { unterer Extremität }\end{array}$ & $\begin{array}{l}\text { Keine explizierte Aus- } \\
\text { wertung bezüglich } \\
\text { katheterassoziierter } \\
\text { Thrombosen }\end{array}$ \\
\hline $\begin{array}{l}\text { Thornburg } \\
\text { et al. [22] }\end{array}$ & $\begin{array}{l}\text { Association between } \\
\text { thrombosis and blood- } \\
\text { stream infection in } \\
\text { neonates with PICC }\end{array}$ & 2007 & $\begin{array}{l}\text { Retro- } \\
\text { spektiv }\end{array}$ & 1540 & $\begin{array}{l}\text { Zusammenhang zwischen } \\
\text { dem Auftreten von Kathe- } \\
\text { terinfektionen und kathe- } \\
\text { terassoziierten Thrombo- } \\
\text { sen }\end{array}$ & $\begin{array}{l}\text { Weitere Untersu- } \\
\text { chungen bzgl. der } \\
\text { Pathogenese not- } \\
\text { wendig }\end{array}$ & $\begin{array}{l}\text { Assoziation zwischen } \\
\text { katheterassoziierten } \\
\text { Thrombosen und } \\
\text { katheterassoziierten } \\
\text { Infektionen darstellbar }\end{array}$ \\
\hline $\begin{array}{l}\text { Tsai et al. } \\
{[23]}\end{array}$ & $\begin{array}{l}\text { Complication rates with } \\
\text { central venous catheters } \\
\text { inserted at femoral und } \\
\text { non-femoral sites in } \\
\text { very low birth weight } \\
\text { infants }\end{array}$ & 2009 & $\begin{array}{l}\text { Retro- } \\
\text { spektiv }\end{array}$ & 518 & $\begin{array}{l}\text { Häufiger katheterassozi- } \\
\text { ierte Sepsis bei PICC von } \\
\text { unterer Extremität, häufi- } \\
\text { ger Thrombophlebitis und } \\
\text { Dislokation bei PICC von } \\
\text { oberer Extremität }\end{array}$ & $\begin{array}{l}\text { Wenn möglich, keine } \\
\text { Anlage von unterer } \\
\text { Extremität aufgrund } \\
\text { häufigerer kathe- } \\
\text { terassoziierter Sepses }\end{array}$ & $\begin{array}{l}\text { Inzidenz von Throm- } \\
\text { bose vermutlich un- } \\
\text { terschätzt, da häufig } \\
\text { asymptomatisch, } \\
\text { etwas weniger Throm- } \\
\text { bosen von der unteren } \\
\text { Extremität }\end{array}$ \\
\hline $\begin{array}{l}\text { Wrightson } \\
{[24]}\end{array}$ & $\begin{array}{l}\text { Peripherally inserted } \\
\text { central catheter com- } \\
\text { plications in neonates } \\
\text { with upper versus lower } \\
\text { extremity insertion sites }\end{array}$ & 2013 & $\begin{array}{l}\text { Retro- } \\
\text { spektiv }\end{array}$ & 626 & $\begin{array}{l}\text { Kein signifikanter Unter- } \\
\text { schied in Komplikationen } \\
\text { zwischen oberem und } \\
\text { unterem Zugangsweg, } \\
\text { häufiger dezentrale Lage } \\
\text { der Katheterspitze bei PICC } \\
\text { von oberer Extremität }\end{array}$ & $\begin{array}{l}\text { Auf zentrale Lage des } \\
\text { Katheters achten um } \\
\text { Komplikationen zu } \\
\text { vermeiden }\end{array}$ & $\begin{array}{l}\text { Keine explizierte Aus- } \\
\text { wertung bezüglich } \\
\text { katheterassoziierter } \\
\text { Thrombosen }\end{array}$ \\
\hline
\end{tabular}

Um einen signifikanten Zusammenhang zwischen klinischem Verlauf, Symptomen und Risikofaktoren für das Entstehen bzw. Vorliegen katheterassoziierter Thrombosen zu erkennen, sind systematische, prospektive Beobachtungsstudien (ggf. mit Vergleichskollektiv) notwendig. Dies wäre auch zur Beantwortung der Frage nach Inzidenz und zu präferierendem Zugangsweg für die Katheteranlage hilfreich [12]. Ob regelmäßige sonogra- phische Screeninguntersuchung aller Patienten mit einliegendem Katheter sinnvoll wären und umzusetzen sind („,minimal handling"), bleibt zu diskutieren.

\section{Limitationen}

Unsere Betrachtung konzentrierte sich ausschließlich auf die Patienten, welche in besagtem Zeitraum eine klinisch auffällige katheterassoziierte Thrombose entwickelt hatten. Bemerkenswerterweise waren hauptsächlich Thrombosen im Stromgebiet der V. cava inferior bei liegendem Katheter von der unteren Extremität detektiert worden und bildeten damit die Grundlage für diese Erhebung. Eine Vergleichsgruppe mit ähnlichem Patientenkollektiv ist retrospektiv nicht zu bilden.

Ob Thrombosen in unserem Studienzeitraum subklinisch verlaufen sind, wur- 
de nicht erhoben. Jedoch stellt sich somit auch die Frage nach einer klinischen Relevanz und therapeutischen Konsequenz.

\section{Fazit für die Praxis}

- Bei Frühgeborenen sind peripher eingeführte zentrale Katheter ein etablierter Zugangsweg zur parenteralen Ernährung und zur Verabreichung von Medikamenten und Flüssigkeit.

- Allein das Vorhandensein eines Katheters begünstigt die Entstehung einer Thrombose.

- Bei klinischen Auffälligkeiten, wie Schwellung der Extremität oder Thrombozytopenie, sollte eine Thrombose am Katheter mittels Sonographie ausgeschlossen werden.

- Frühgeborene eines extrem niedrigen Geburtsgewichtes und Frühgeborene mit einem komplizierten postnatalen Verlauf scheinen ein erhöhtes Risiko für das Entwickeln einer katheterassoziierten Thrombose zu haben.

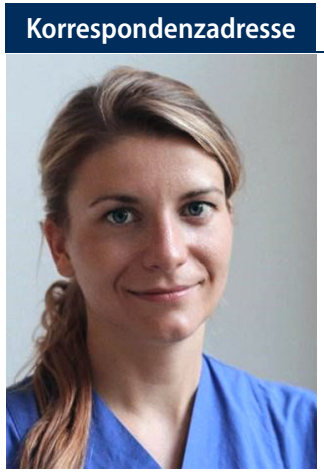

\section{Dr. med. S. Tippmann}

Neonatologie, Zentrum für Kinder - und Jugendmedizin der Unimedizin Mainz Langenbeckstr. 1, 55131 Mainz, Deutschland Susanne.Tippmann@unimedizin-mainz.de

Funding. Open Access funding enabled and organized by Projekt DEAL.

\section{Einhaltung ethischer Richtlinien}

Interessenkonflikt. S. Tippmann, K. Schmitz, C. Whybra, G. Staatz, C. Kampmann und E. Mildenberger geben an, dass kein Interessenkonflikt besteht.

Für diese retrospektive Analyse wurden die ethischen Richtlinien gemäß Landeskrankenhausgesetz $§ 36$, $\S 37$ Rheinland-Pfalz eingehalten.

Open Access. Dieser Artikel wird unter der Creative Commons Namensnennung 4.0 International Lizenz veröffentlicht, welche die Nutzung, Vervielfältigung, Bearbeitung, Verbreitung und Wiedergabe in jegli-

\section{Characteristics and clinical manifestation of catheter-associated thrombosis in premature infants}

Background: In neonatology peripherally inserted central catheters are a frequently used access route for parenteral nutrition and for the administration of drugs and fluids. The advantages are offset by the risks, such as infection, thrombosis and malpositioning.

Objective: Which characteristics and clinical signs indicate the presence of catheterassociated thrombosis?

Material and methods: In a retrospective study all cases of catheter-associated thrombosis from 2010 to 2016 were analyzed and 10 premature infants were identified. Data on indications for catheter placement, access route, position of the catheter tip, length of stay, clinical symptoms and course of disease were collected.

Results: In 10 preterm infants with a gestational age of $23+4-34+5$ weeks 11 thrombotic events were observed. Out of 11 thrombotic events 10 occurred in the inferior vena cava. Indications for catheter placement were parenteral nutrition and surgical interventions. Perinatal complications had occurred in 9 patients. All patients were suspected to have an infection while the catheters were in place. The occurrence of thrombosis was documented after 3-27 days by ultrasound. In 8 thrombotic events thrombocytopenia was conspicuous, in 4 cases leg swelling led to the diagnosis.

Discussion: In our patients, similarities in the clinical course and in the symptoms indicated the presence of thrombosis. Interestingly, most thrombotic events occurred in the inferior vena cava.

\section{Keywords}

Peripherally inserted central catheter · Complications · Thrombosis · Thrombozytopenia · Vena cava inferior

chem Medium und Format erlaubt, sofern Sie den/die ursprünglichen Autor(en) und die Quelle ordnungsgemäß nennen, einen Link zur Creative Commons Lizenz beifügen und angeben, ob Änderungen vorgenommen wurden.

Die in diesem Artikel enthaltenen Bilder und sonstiges Drittmaterial unterliegen ebenfalls der genannten Creative Commons Lizenz, sofern sich aus der Abbildungslegende nichts anderes ergibt. Sofern das betreffende Material nicht unter der genannten Creative Commons Lizenz steht und die betreffende Handlung nicht nach gesetzlichen Vorschriften erlaubt ist, ist für die oben aufgeführten Weiterverwendungen des Materials die Einwilligung des jeweiligen Rechteinhabers einzuholen.

Weitere Details zur Lizenz entnehmen Sie bitte der Lizenzinformation auf http://creativecommons.org/ licenses/by/4.0/deed.de.

\section{Literatur}

1. Bashir RA, Swarnam K, Vayalthrikkovil S, Yee W, Soraisham AS (2016) Association between peripherally inserted central venous catheter insertion site and complication rates in preterm infants. Amer JPerinatol 33(10):945-950

2. Bauman ME, Cheung P-Y, Massicotte MP (2011) Hemostasis and platelet dysfunction in asphyxiated neonates. JPediatr 158(2):e35-e39

3. van den Berg J, Lööf Åström J, Olofsson J, Fridlund M, Farooqi A (2017) Peripherally inserted central catheter in extremely preterm infants: Characteristics and influencing factors. NPM 10(1):63-70
4. Blackwood BP, Farrow KN, Kim S, Hunter CJ (2016) Peripherally inserted central catheters complicated by vascular erosion in neonates. JPEN JParenter Enteral Nutr 40(6):890-895

5. Bradford NK, Edwards RM, Chan RJ (2016) Heparin versus $0.9 \%$ sodium chloride intermittent flushing for the prevention of occlusion in long term central venous catheters in infants and children: a systematic review. Int J Nurs Stud 59:51-59

6. Bulbul A, Okan F, Nuhoglu A (2010) Percutaneously inserted central catheters in the newborns: a center's experience in Turkey. J Matern Neonatal Med 23(6):529-535

7. Christoph J, Dame C, Geffers C (2018) Prävention von Gefäßkatheter-assoziierten Infektionen bei Früh- und Neugeborenen Empfehlung der Kommission für Krankenhaushygiene und Infektionsprävention (KRINKO) beim Robert KochInstitut. Bundesgesundheitsblatt 61:608-626. https://doi.org/10.1007/s00103-018-2718-y

8. Costa P, Kimura Fumiko A, Huffman BD, Damiani LP (2016) Predictors of nonelective removal of peripherally inserted central catheters in infants. Biol Res Nurs 18(2):173-180

9. Cushman M (2005) Inherited risk factors for venous thrombosis. Hematol Am Soc Hematol Educ Program 452-457. https://doi.org/10.1182/ asheducation-2005.1.452

10. Edwards JR, Peterson KD, Mu Y, Banerjee S, AllenBridson K, Morrell G, Dudeck MA, Pollock DA et al (2009) National Healthcare Safety Network (NHSN) report: data summary for 2006 through 2008, issued December 2009. Am J Infect Control 37(10):783-805

11. Geffers C, Kramer A, Scheithauer (2017) Prävention von Infektionen, die von Gefäßkathetern ausgehenTeil 1 - Nichtgetunnelte zentralvenöse KatheterEmpfehlung derKommission für Kranken- 


\section{Originalien}

haushygiene und Infektionsprävention (KRINKO) beim Robert Koch-Institut. Bundesgesundheitsblatt 60:171-206

12. Gomez Azedavedo BF (2013) Complications of the central catheters in a level III neonatal intensive care unit Bd. Mestrado Inegrado Med 32(12):941-6. 10.1038/jp.2012.7

13. Hoang V, Sills J, Chandler M, Busalani E, CliftonKoeppel R, Modanlou HD (2008) Percutaneously inserted central catheter for total parenteral nutrition in neonates: complications rates related to upper versus lower extremity insertion. Pediatrics 121(5):e1152-1159

14. Isemann B, Sorrels R, Akinbi H (2012) Effect of heparin and other factors associated with complications of peripherally inserted central venous catheters in neonates. J Perinatol 32(11):856-860

15. Jumani K, Advani S, Reich NG, Gosey L, Milstone AM (2013) Risk factors for peripherally inserted central venous catheter complications in children. JAMA Pediatr 167(5):429-435

16. Kisa P, Ting J, Callejas A, Osiovich $H$, Butterworth SA (2015) Major thrombotic complications with lower limb PICCs in surgical neonates. J Pediatr Surg 50(5):786-789

17. Monagle P, Chan AKC, Goldenberg NA, Ichord RN, Journeycake JM, Nowak-Göttl U, Vesely SK (2012) Antithrombotic therapy in neonates and children: antithrombotic therapy and prevention of thrombosis, 9th ed: American college of chest physicians evidence-based clinical practice guidelines. Chest 141(2):e737S-e801S

18. Njere I, Saidul I, Parish D, Jauro K, Keshtgar, Alireza S (2011) Outcome of peripherally inserted central venous catheters in surgical and medical neonates. JPediatr Surg 46(5):946-950

19. Ohki Y, Maruyama K, Harigaya A, Kohno M, Arakawa H (2013) Complications of peripherally inserted central venous catheter in Japanese neonatal intensive care units. Pediatr Int 55(2):185-189

20. Ozkiraz S, Gokmen Z, Anuk Ince D, Akcan Baris A, Kilicdag H, Ozel D, Ecevit A (2013) Peripherally inserted central venous catheters in critically ill premature neonates. JVasc Access 14(4):320-324

21. Park CK, Paes BA, Nagel K, Chan AK, Murthy $P$, Thrombosis and Hemostasis in Newborns (THiN) Group (2014) Neonatal central venous catheter thrombosis: diagnosis, management and outcome. Blood Coagul Fibrinol 25(2):97-106

22. Pettit J (2002) Assessment of infants with peripherally inserted central catheters: part 1. Detecting the most frequently occurring complications. Adv Neonatal Care 2(6):304-315

23. Pettit J (2003) Assessment of infants with peripherally inserted central catheters: Part 2. Detecting less frequently occurring complications. Adv Neonatal Care 3(1):14-26

24. Smitherman $A B$, Alexander $T$, Connelly $M$, Snavely AC, Weston BW, Liles EA, Steiner MJ (2015) The incidence of catheter-associated venous thrombosis in noncritically ill children. Hosp Pediatr 5(2):59-66

25. Thornburg CD, Smith PB, Smithwick ML, Cotten CM, Benjamin DK (2008) Association between thrombosis and bloodstream infection in neonates with peripherally inserted catheters. Thromb Res 122(6):782-785

26. Tsai $M-H$, Reyin L, Jiunn-Wei W, Hsuan-Rong $H$, Chiao-Ching C, Chu S-M, Hsu J-F, Yhu-Chering H (2009) Complication rates with central venous catheters inserted at femoral and non-femoral sites in very low birth weight infants. Pediatr Infect Dis J 28(11):966-970
27. Wrightson Daugherty D (2013) Peripherally inserted central catheter complications in neonates with upper versus lower extremity insertion sites. Adv Neonatal Care 13(3):198-204 